Open Access

\title{
Preoperative Controlling Nutritional Status (CONUT) score predicts short-term outcomes of patients with gastric cancer after laparoscopy-assisted radical gastrectomy
}

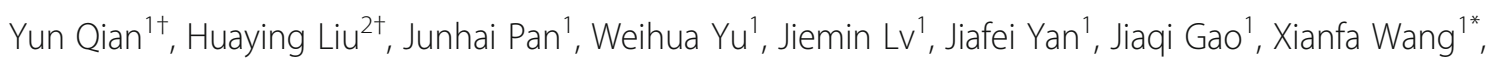
Xiaolong $\mathrm{Ge}^{1^{*}}$ (D) and Wei Zhou ${ }^{1}$

\begin{abstract}
Background: An emerging prediction tool, the Controlling Nutritional Status (CONUT) score, has shown good assessment ability of postoperative outcomes in cancer patients. This study evaluated the role of the preoperative CONUT score regarding the short-term outcomes of gastric cancer (GC) after laparoscopic gastrectomy.

Methods: Three hundred and nine GC patients undergoing laparoscopic gastrectomy from January 2016 to June 2019 were analysed, retrospectively. The patients were divided into two groups according to the CONUT optimal cut-off value. Clinical characteristics and postoperative complications in the two groups were analysed and evaluated. Risk factors for complications were identified by univariate and multivariate analyses.
\end{abstract}

Results: A total of 309 patients underwent laparoscopic gastrectomy; 91 (29.4\%) patients experienced postoperative complications. The preoperative CONUT score showed a good predictive ability for postoperative complications (area under the curve $(A \cup C)=0.718$, Youden index $=0.343$ ) compared with other indices, with an optimal cut-off value of 2.5. Patients with high CONUT score had a significantly higher incidence of overall complications $(P<$ 0.001). Age, haemoglobin, C-reactive protein, red blood cell levels, CONUT scores, surgical procedure type, T1, T4, NO and N3 pathological TNM classification, and pathological stages of I and III were associated with postoperative complications $(P<0.05)$. Furthermore, the preoperative CONUT score was identified as an independent risk predictor of postoperative complications $(P=0.012 ; \mathrm{OR}=2.433 ; 95 \% \mathrm{Cl}, 1.218-4.862)$ after multivariate analysis.

Conclusions: The preoperative CONUT score is a practical nutritional assessment for predicting short-term outcomes in GC patients after laparoscopy-assisted gastrectomy.

Keywords: Controlling Nutritional Status score, Gastric cancer, Postoperative complications, Laparoscopic surgery

\footnotetext{
* Correspondence: 3195011@zju.edu.cn; gxlmed@zju.edu.cn

${ }^{\dagger}$ Yun Qian and Huaying Liu contributed equally to this work.

'Department of General Surgery, Sir Run Run Shaw Hospital, School of

Medicine, Zhejiang University, 3 East Qingchun Road, Hangzhou 310016,

China

Full list of author information is available at the end of the article
}

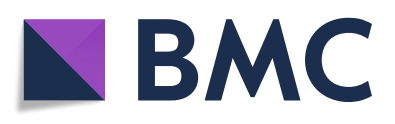

(- The Author(s). 2021 Open Access This article is licensed under a Creative Commons Attribution 4.0 International License, which permits use, sharing, adaptation, distribution and reproduction in any medium or format, as long as you give appropriate credit to the original author(s) and the source, provide a link to the Creative Commons licence, and indicate if changes were made. The images or other third party material in this article are included in the article's Creative Commons licence, unless indicated otherwise in a credit line to the material. If material is not included in the article's Creative Commons licence and your intended use is not permitted by statutory regulation or exceeds the permitted use, you will need to obtain permission directly from the copyright holder. To view a copy of this licence, visit http://creativecommons.org/licenses/by/4.0/. The Creative Commons Public Domain Dedication waiver (http://creativecommons.org/publicdomain/zero/1.0/) applies to the data made available in this article, unless otherwise stated in a credit line to the data. 


\section{Introduction}

As a major public health issue globally, gastric cancer (GC) is the third leading cause of cancer-related death [1]. Despite recent progress in the diagnosis and treatment of GC, patient prognosis remains poor. The main curative therapeutic option for GC is surgical resection $[2,3]$, with inevitable postoperative complications, leading to longer hospitalisation, greater expenses, poor quality of life and adjuvant chemotherapy therapy delay.

Patients with GC may have to endure unpleasant symptoms, such as early satiety, anorexia and dysphagia, caused by obstruction due to the tumour mass and chronic anaemia due to malignant ulcers. These factors result in progressive weight loss, compromised immunity and ultimately malnutrition [4]. Indeed, malnutrition is quite common and severe amongst patients with GC, especially in those with advanced GC.

Therefore, multiple nutritional assessment systems have emerged with the aim of identifying applicable parameters or tools, detecting malnutrition and predicting outcomes of patients with GC. For example, Oh et al. [5] analysed patients with GC and confirmed various perioperative nutritional parameters, including the prognostic nutritional index (PNI) and albumin (ALB), to be independent predictors of complications. Sun et al. [6] reported that ALB and neutrophils could predict postoperative overall survival (OS) in patients with GC, and Kim et al. [7] observed that the platelet-to-lymphocyte ratio (PLR) is able to predict the prognosis of GC. Other nutritional assessment tools have been reported for cancer patients, including the Nutritional Risk Screening (NRS), Skeletal Muscle Index (SMI) and Naples Prognostic Score (NPS) [8-12].

The Controlling Nutritional Status (CONUT) score was initially reported in 2005 as a useful assessment for the early detection and persistent monitoring of malnutrition [13]. The score consists of serum ALB, total lymphocyte count and cholesterol levels measurements. In recent years, several studies have shown that the CONUT score is a validated and useful assessment of nutritional status for predicting multiple cancer outcomes after surgery, including in colorectal cancer [14], hepatocellular carcinoma [15], oesophageal cancer [16], and GC [8, 17-21]. However, there was few research on the CONUT score in predicting postoperative outcomes in GC patients after radical gastrectomy. Therefore, this study aimed to assess the predictive ability of the preoperative CONUT score with regard to short-term outcomes in GC patients who underwent laparoscopic radical gastrectomy.

\section{Patients and methods}

\section{Study patients}

Consecutive clinical records for 412 patients undergoing laparoscopic gastrectomy from January 2016 to June
2019 were initially examined in this study. The inclusion criteria were as follows: (1) gastric carcinoma confirmed by pathological diagnosis from gastroscopic biopsy, (2) curative laparoscopic gastrectomy performed and (3) age $>18$ years. The exclusion criteria were as follows: (1) neoadjuvant chemotherapy before gastrectomy, (2) R1/2 resection, (3) diagnosed with gastric stump cancer, (4) GC combined with distant metastasis (liver, colon, ovary, etc.), (5) extended or palliative surgery performed and (6) incomplete data during follow-up. Ultimately, 309 patients were enrolled in the retrospective analysis. The detailed flow-chart is shown in Fig. 1. Written informed consent for the usage of clinical records was granted by each patient, as required by the Institutional Review Board at the hospital, in accordance with the ethical guidelines of the Declaration of Helsinki in 1964.

\section{Perioperative management}

Routine case history collection, physical examination, and preoperative laboratory measurements were performed. Abdominal enhanced computed tomography and endoscopy together with tissue biopsy were carried out for the overall assessment of gastric tumours. Standard surgical laparoscopic gastrectomy with a sufficient resection margin was performed according to guidelines [3], which involved either total or distal gastrectomy coupled with systematic lymphadenectomy abiding by $\mathrm{D}$ level criteria. The following alimentary tract reconstruction methods were usually employed: Roux-en-Y esophagojejunostomy was performed after total gastrectomy, whereas Billroth I, Billroth II or Roux-en-Y gastrojejunostomy was selected after distal gastrectomy. For all patients, reasonable perioperative management was in line with the Enhanced Recovery After Surgery (ERAS) programme, including preoperative disease education, reducing fasting time, intraoperative use of minimally invasive techniques, fluid restriction to avoid overload, postoperative early drain removal, off-bed mobilisation and oral feeding until discharge [22-24]. Thereafter, those diagnosed with advanced gastric carcinoma were recommended to receive subsequent adjuvant chemotherapy.

\section{Data collection}

Clinical records of baseline characteristics, laboratory data, imaging scanning examinations and pathological diagnosis were collected from a database. The CONUT score was assessed according to Table 1 . PNI $=10 \times$ serum ALB $(\mathrm{g} / \mathrm{dL})+0.005 \times$ total lymphocyte count $($ per $\mathrm{mm}^{3}$ ), and PLR $=$ platelet count/total lymphocyte count. Short-term outcomes were mainly postoperative complications that occurred within 30 days after laparoscopic surgery or before hospital discharge.

According to the Clavien-Dindo classification system [25], mild complications included grades I and II and major 


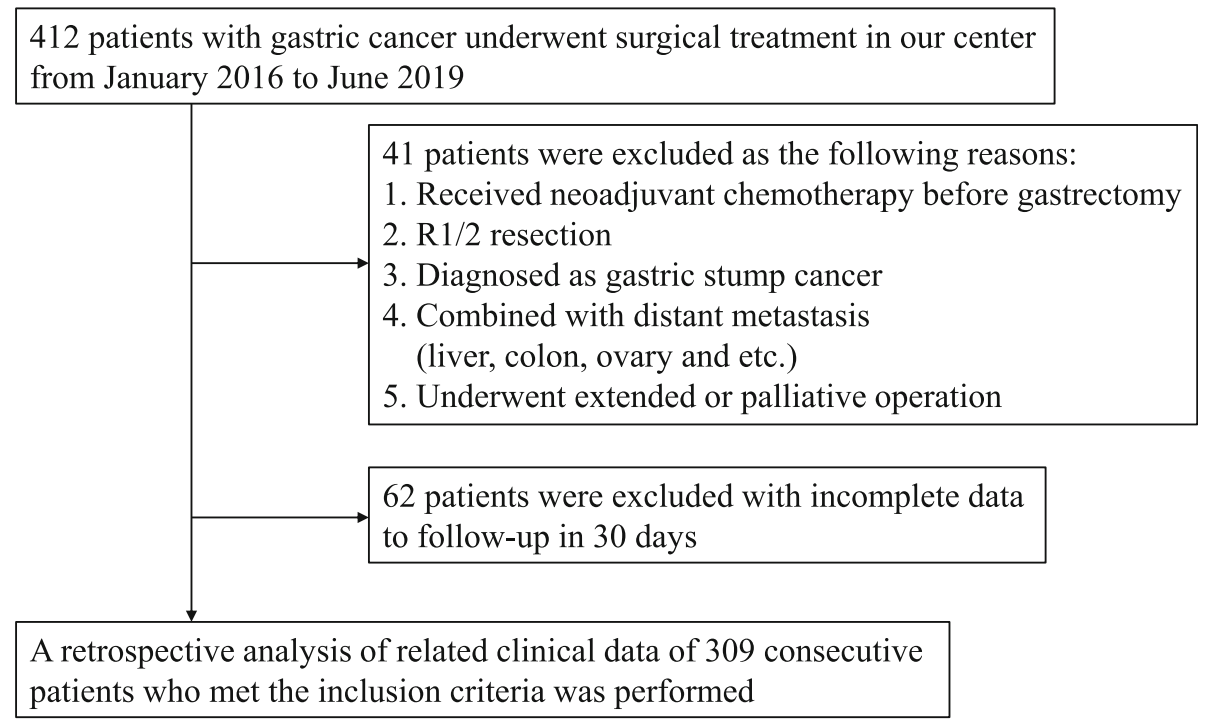

Fig. $1 \mathrm{~A}$ flow chart of the inclusion process for patients with gastric cancer

complications included grades III to IV as previously described [26]. For major complications, severe active haemorrhage after surgery required emergency treatment. When persistent fever and purulent drainage occurred, an internal intra-abdominal abscess was considered. Other major intractable complications included anastomotic leakage and duodenal stump fistula. Other postoperative events included respiratory complications, cardiovascular complications and surgical site infections (SSIs) [27]. Data on cancer staging were evaluated based on the Tumour-NodeMetastasis (TNM) Classification of malignant tumour.

\section{Statistical analysis}

Data were statistically analysed using SPSS 23.0. Quantitative variables are presented as the mean $\pm \mathrm{SD}$; qualitative variables are presented as numbers (percentages). Student's $t$ test or the Mann-Whitney $U$ test was utilised for quantitative data. The Pearson $\chi^{2}$ test was applied for qualitative data. Receiver operating characteristic (ROC)

Table 1 Assessment of malnutritional status by the CONUT score

\begin{tabular}{lllll}
\hline Parameter & \multicolumn{4}{l}{ Malnutritional status } \\
\cline { 2 - 5 } & Normal & Mild & Moderate & Severe \\
\hline ALB $(\mathrm{g} / \mathrm{dl})$ & $\geq 3.5$ & $3.0 \leq \mathrm{ALB}<3.5$ & $2.5 \leq \mathrm{ALB}<3.0$ & $<2.5$ \\
Score & 0 & 2 & 4 & 6 \\
$\mathrm{TLC}(\mathrm{mg} / \mathrm{ml})$ & $\geq 1600$ & $1200 \leq \mathrm{TLC}<1600$ & $800 \leq \mathrm{ALB}<1200$ & $<800$ \\
Score & 0 & 1 & 2 & 3 \\
TC $(\mathrm{mg} / \mathrm{dl})$ & $\geq 180$ & $140 \leq \mathrm{TC}<180$ & $100 \leq \mathrm{TC}<140$ & $<100$ \\
Score & 0 & 1 & 2 & 3 \\
Total score & $0-1$ & $2-4$ & $5-8$ & $9-12$ \\
\hline
\end{tabular}

$A L B$ albumin; $T L C$ total lymphocyte count; $T C$ total cholesterol curve analysis was performed to analyse the predictive ability of factors, including the CONUT score, PNI, ALB and PLR. To identify independent risk predictors for postoperative complications, factors with $P$ values less than 0.05 in univariate analysis were assessed in multivariate analysis. The indicators of serum ALB, total lymphocyte count and cholesterol were excluded from multivariate analysis to avoid duplication. Significance was defined as $P$ values less than 0.05 .

\section{Results}

ROC curve of the CONUT score, PNI, ALB and PLR

In total, 309 patients were enrolled in this study. The ROC curves of the CONUT score, PNI, ALB and PLR were drawn, and the areas under the curve (AUC) were $0.718,0.694,0.680$ and 0.635 , respectively (Fig. 2). The CONUT score was the most useful predictor. The demarcated values of the CONUT score that correlated with outcomes differed from those in previous studies $[8,14-19]$. In our study, the cut-off value in the prediction of postoperative complications was identified as 2.5. The Youden index of the CONUT score was 0.343, with a sensitivity of 0.549 and specificity of 0.794 . The positive predictive value for postoperative complications was $52.3 \%$; the negative predictive value was $80.8 \%$.

\section{Study population and baseline characteristics based on the CONUT score cut-off value}

According to the cut-off value of the CONUT score, 214 patients with scores less than 2.5 were allocated into the low CONUT score group; 95 patients with scores greater than 2.5 were allocated into the high CONUT score group. The average age of low CONUT score group 
A

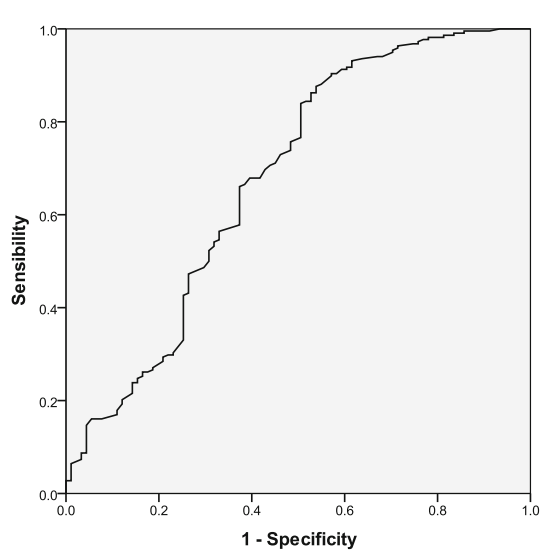

C

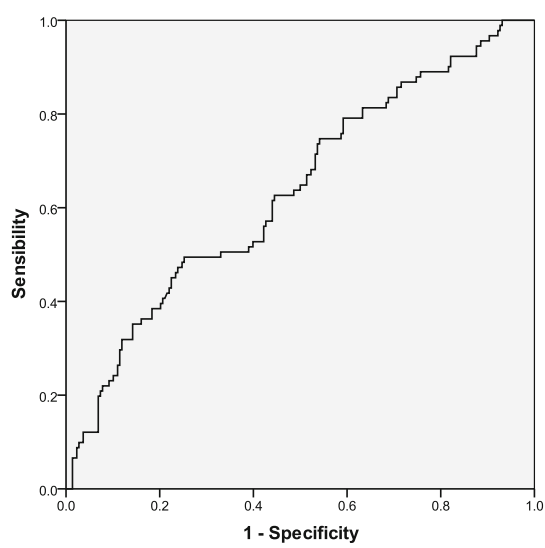

B

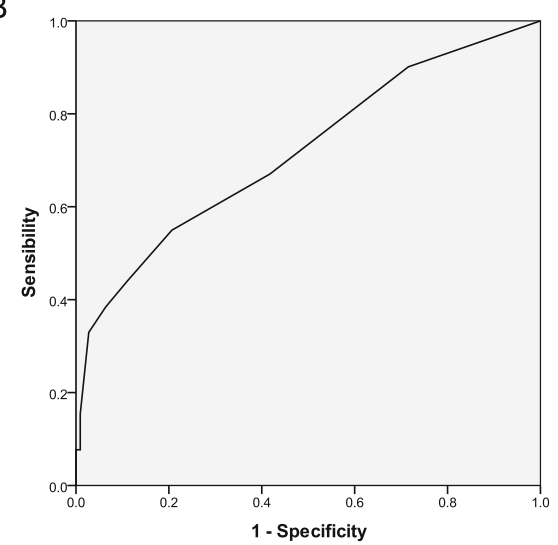

D

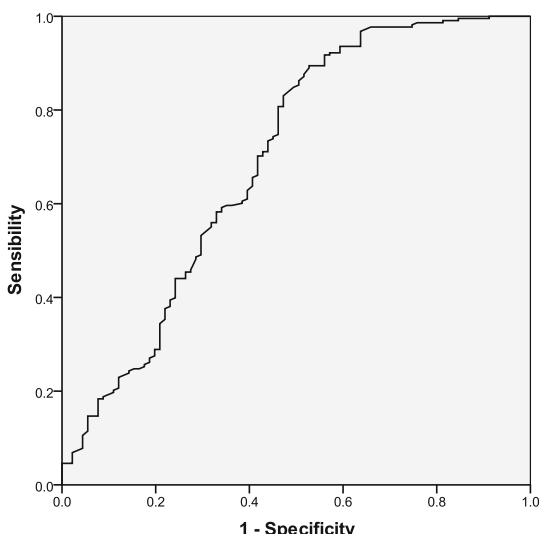

Fig. 2 Receiver operating characteristic curve showing the capacity of ALB (a), CONUT score (b), PLR (c) and PNI (d) for predicting postoperative overall complications

patients was much lower than that of high score group patients $(62.2 \pm 0.7$ years vs $66.2 \pm 1.2$ years, $P=0.003)$. The ratio of males to females in the two groups was not significantly different ( $155 / 59$ vs $73 / 22, P=0.416)$. Overall, body mass index (BMI) was significantly lower in patients with a CONUT score $>2.5$ than in those with a CONUT score $<2.5\left(21.9 \pm 0.3 \mathrm{~kg} / \mathrm{m}^{2}\right.$ vs $\left.23.1 \pm 0.2 \mathrm{~kg} / \mathrm{m}^{2}, P<0.001\right)$. The rate of diabetes mellitus was significantly higher in the high CONUT score group ( $8.9 \%$ vs $20.0 \%, P=0.006$ ), though no significant difference in hypertension was found $(35.5 \%$ vs $43.2 \%, P=0.201)$. The high CONUT score group had undergone much more previous abdominal surgery $(18.7 \%$ vs $31.6 \%, P=0.013)$. There were 193 (62.5\%) patients who received distal gastrectomy and 116 (37.5\%) who received total gastrectomy, with no significant difference between those with high and low scores (131/83 vs $62 / 33, P=0.498)$.

\section{Comparison of clinical characteristics between patients with low and high CONUT scores}

Compared to the low CONUT score group, the levels of preoperative haemoglobin $(\mathrm{Hb})(132.5 \pm 1.3$ vs $104.5 \pm$
2.4, $P<0.001)$, ALB $(40.9 \pm 0.3$ vs $34.6 \pm 0.5, P<$ $0.001)$, red blood cells (RBCs) $(4.38 \pm 0.04$ vs $3.60 \pm$ $0.07, P<0.001)$, platelets (PLTs) $(221.4 \pm 4.5$ vs 206.5 $\pm 7.4, P<0.001)$, total lymphocytes $(1.79 \pm 0.04 \mathrm{vs}$ $1.04 \pm 0.04, P<0.001)$ and cholesterol $(5.02 \pm 0.07 \mathrm{vs}$ $3.64 \pm 0.08, P<0.001)$ were lower, and the $C$-reactive protein $(\mathrm{CRP})$ level $(3.2 \pm 0.6$ vs $9.6 \pm 1.8, P<0.001)$ were higher in the high CONUT score group. Regarding preoperative tumour biomarkers, there were significant differences in carbohydrate antigen 125 (CA125) $(P=0.001)$ and carbohydrate antigen 199 (CA199) $(P=0.016)$ but not in carcinoembryonic antigen $(\mathrm{CEA})(P=0.769)$ or alpha fetoprotein (AFP) $(P=$ 0.487). Patients with low CONUT scores were more likely to have pathological stage I disease $(37.4 \%$ vs $23.2 \%, P=0.014)$; patients with high CONUT scores were more likely to have stage III disease. The high CONUT score group experienced significantly more postoperative complications $(19.2 \%$ vs $52.6 \%, P<0.001)$ and had a longer postoperative stay $(11.6 \pm 0.5$ days vs $14.1 \pm 0.7$ days, $P=0.006$ ) than the low CONUT score group. More details are shown in Table 2 . 


\section{Postoperative complications in GC patients with low and high CONUT scores}

The rate of postoperative complications in patients with a CONUT score $<2.5$ was significantly lower than that in patients with a CONUT score $>2.5(19.2 \%$ vs $52.6 \%$, $P<0.001$ ) (Table 3$)$. The rate of mild complications, including sustained fever with a temperature over $38.5^{\circ} \mathrm{C}$, incision infection, persistent utilisation of total parenteral nutrition exceeding 2 weeks, postoperative blood transfusion, gastroplegia, abdominal or pelvic effusion, early postoperative bowel obstruction and urinary tract infection, was significantly higher in the high CONUT score group (8.4\% vs $34.7 \%, P<0.001)$. A total of 40 patients developed major complications, including postoperative active haemorrhage, intra-abdominal abscess, anastomotic leakage, duodenal stump fistula, septic shock and single organ dysfunction, and there was a significant difference between the two groups $(18.7 \%$ vs $31.6 \%, P=0.013$ ) (Table 3 ). Only 1 patient died after surgery, from severe cachexia and multiple organ dysfunction syndrome (MODS). With regard to SSIs, there were $5(1.6 \%)$ cases of surface incisional infection and 20 (6.5\%) cases of deep space infection, with no significant difference between the two groups $(0.9 \%$ vs $3.2 \%, P=$ $0.347 ; 5.1 \%$ vs $9.5 \%, P=0.153$ ).

\section{Univariate and multivariate analyses of risk factors for short-term outcomes in GC}

In univariate analysis, age, Hb, CRP, RBCs, CONUT score, type of operative procedure, pathological TNM classification of T1, T4, N0 and N3, and pathological stage of I and III were found to be risk factors with a $P$ value less than 0.05 . Furthermore, age $(P=0.037$; odds ratio $(\mathrm{OR})=2.237$; $95 \%$ confidence interval $(\mathrm{CI}), 1.048$ 4.774), RBCs $(P=0.003$; OR $=0.356$; $95 \%$ CI, 0.180 0.707), and CONUT scores $(P=0.012$; OR $=2.433 ; 95 \%$ $\mathrm{CI}, 1.218-4.862)$ were identified as independent risk indicators for postoperative complications in GC after laparoscopic gastrectomy (Table 4).

\section{Discussion}

A clinical database with a consecutive patient cohort was analysed to explain whether the preoperative CONUT score is able to effectively predict postoperative complications for GC patients after laparoscopic gastrectomy. This study found that the preoperative CONUT score is an independent risk factor for predicting postoperative complications in GC after surgery.

The prognosis of cancer is not only associated with tumour factors but also with patient status, especially nutritional status [28, 29]. The CONUT score was originally proposed by Ignacio de Ulibarri J in 2005 as an integrated scale for assessing the nutritional status of inpatients [13]. The CONUT score can reflect protein reserves, immune function and lipid metabolism. The condition of hypoalbuminaemia suggests that the body is in a stage of hypercatabolism, which is prevalent amongst cancer patients, especially those with cachexia. Lymphocytes are important cellular components of the human immune response system that help to fight tumours by inhibiting cancer cell proliferation, invasion and migration [30]. Saka et al. [31] reported that $\mathrm{T}$ cell exhaustion was closely associated with poor prognosis in cancer. Cholesterol plays a vital role in modulating the activity of membrane proteins, which may be associated with the occurrence and development of cancer and interactions with the body's immune system. Additionally, Yang et al. [32] reported that cholesterol inhibited hepatocellular carcinoma invasion and metastasis by promoting CD44 localization in lipid rafts. Therefore, this assessment scale is able to provide an integrated, rapid, and low-cost nutritional evaluation of patients.

Previous studies have proposed diversified prognostic predictors for GC, such as the PNI [5, 18, 33-36] and PLR [7, 37, 38]. These nutritional score scales are based on routine parameters from blood examinations and are applied to assess the prognosis of cancer patients. In our study, we analysed the assessment capability of these scales for predicting postoperative complications with ROC curves, and the CONUT score showed the best performance. The cut-off value for CONUT in our study was 2.5 , which was in line with previous studies [18-20]. For example, Hirahara et al. [19] compared the prognostic value of the CONUT score with low $(\leq 2)$ and high $(\geq 3)$ score with propensity score matching in patients with gastrectomy. Liu's study involved 697 consecutive patients for stage II-III gastric cancer and concluded that the high CONUT group $(\geq 3)$ had a significantly lower 5-year survival [20]. There was also different optimal threshold of CONUT score in other studies. For example, Ryo et al. [17] determined the cut-off value of CONUT score as 2 for predicting mortality in 2 years and overall survival. Although the optimal value was different, the CONUT score still showed a good correlation with the outcomes of GC patients $[17,39]$. In addition, we identified age and RBC count as independent risk factors for complications. In other words, old age, anaemia and malnutrition had an adverse effect on short-term outcomes in patients after gastrectomy for GC, which was consistent with prior studies $[39,40]$.

In previous studies, most researchers have focused on long-term survival associated with the CONUT score amongst GC patients [8,17-21], with some focus on postoperative complications. Ryo et al. [17] mentioned the incidence of some complications, such as 
Table 2 Study population and baseline characteristics of the patients sorted by the CONUT score

\begin{tabular}{|c|c|c|c|c|}
\hline Characteristics & All $(N=309)$ & CONUT $<2.5(N=214)$ & CONUT > $2.5(N=95)$ & $P$ value \\
\hline Age, years & $63.4 \pm 0.6$ & $62.2 \pm 0.7$ & $66.2 \pm 1.2$ & 0.003 \\
\hline Gender & & & & 0.416 \\
\hline Male & $228(73.8)$ & $155(72.4)$ & $73(76.8)$ & \\
\hline Female & $81(26.2)$ & $59(27.6)$ & $22(23.2)$ & \\
\hline $\mathrm{BMI}, \mathrm{kg} / \mathrm{m}^{2}$ & $22.8 \pm 0.2$ & $23.1 \pm 0.2$ & $21.9 \pm 0.3$ & $<0.001$ \\
\hline \multicolumn{5}{|l|}{ Comorbidities } \\
\hline Diabetes mellitus & $38(12.3)$ & $19(8.9)$ & $19(20.0)$ & 0.006 \\
\hline Hypertension & $117(37.9)$ & $76(35.5)$ & $41(43.2)$ & 0.201 \\
\hline History of abdomen surgery & $70(22.7)$ & $40(18.7)$ & $30(31.6)$ & 0.013 \\
\hline \multicolumn{5}{|c|}{ Preoperative laboratory measurements } \\
\hline $\mathrm{Hb}, \mathrm{g} / \mathrm{L}$ & $123.9 \pm 1.4$ & $132.5 \pm 1.3$ & $104.5 \pm 2.4$ & $<0.001$ \\
\hline Albumin, g/L & $39.0 \pm 0.3$ & $40.9 \pm 0.3$ & $34.6 \pm 0.5$ & $<0.001$ \\
\hline CRP, mg/L & $5.2 \pm 0.7$ & $3.2 \pm 0.6$ & $9.6 \pm 1.8$ & $<0.001$ \\
\hline $\mathrm{WBC}, \times 10^{9} / \mathrm{L}$ & $5.87 \pm 0.09$ & $5.99 \pm 0.10$ & $5.61 \pm 0.21$ & 0.064 \\
\hline $\mathrm{RBC}, \times 10^{12} / \mathrm{L}$ & $4.14 \pm 0.04$ & $4.38 \pm 0.04$ & $3.60 \pm 0.07$ & $<0.001$ \\
\hline Platelets, $\times 10^{9} / \mathrm{L}$ & $216.8 \pm 3.8$ & $221.4 \pm 4.5$ & $206.5 \pm 7.4$ & $<0.001$ \\
\hline Total lymphocytes, $\times 10^{9} / \mathrm{L}$ & $1.56 \pm 0.04$ & $1.79 \pm 0.04$ & $1.04 \pm 0.04$ & $<0.001$ \\
\hline Cholesterol, mmol/L & $4.60 \pm 0.06$ & $5.02 \pm 0.07$ & $3.64 \pm 0.08$ & $<0.001$ \\
\hline \multicolumn{5}{|l|}{ Preoperative tumour biomarkers } \\
\hline $\mathrm{CA} 125, \mathrm{u} / \mathrm{ml}$ & $13.6 \pm 0.8$ & $11.8 \pm 0.9$ & $17.4 \pm 1.7$ & 0.001 \\
\hline CA199, u/ml & $27.9 \pm 4.6$ & $20.4 \pm 2.8$ & $43.9 \pm 13.0$ & 0.016 \\
\hline CEA, ng/ml & $5.8 \pm 1.6$ & $6.1 \pm 2.3$ & $5.1 \pm 1.2$ & 0.769 \\
\hline AFP, $\mu \mathrm{g} / \mathrm{L}$ & $8.9 \pm 5.8$ & $11.7 \pm 8.4$ & $3.0 \pm 0.5$ & 0.487 \\
\hline Types of operative procedure & & & & 0.498 \\
\hline Distal gastrectomy & $193(62.5)$ & $131(61.2)$ & $62(65.3)$ & \\
\hline Total gastrectomy & $116(37.5)$ & $83(38.8)$ & $33(34.7)$ & \\
\hline Intraoperative fluid utilisation, $\mathrm{ml}$ & $2161 \pm 35.7$ & $2209 \pm 41.1$ & $2042 \pm 69.4$ & 0.034 \\
\hline Operative time, min & $271.8 \pm 3.0$ & $273.4 \pm 3.6$ & $268.4 \pm 5.8$ & 0.444 \\
\hline Estimated blood loss, ml & $106.1 \pm 7.3$ & $103.0 \pm 9.3$ & $112.9 \pm 11.2$ & 0.531 \\
\hline \multicolumn{5}{|l|}{ T factor } \\
\hline $\mathrm{T} 1$ & $91(29.4)$ & $78(36.4)$ & $13(13.7)$ & $<0.001$ \\
\hline $\mathrm{T} 2$ & $35(11.3)$ & $22(10.3)$ & $13(13.7)$ & 0.384 \\
\hline T3 & $42(13.6)$ & $23(10.7)$ & $19(20.0)$ & 0.029 \\
\hline T4 & $141(45.6)$ & $91(42.5)$ & $50(52.6)$ & 0.100 \\
\hline \multicolumn{5}{|l|}{$\mathrm{N}$ factor } \\
\hline No & $118(38.2)$ & $94(43.9)$ & $24(25.3)$ & 0.002 \\
\hline $\mathrm{N} 1$ & $44(14.2)$ & $30(14.0)$ & $14(14.7)$ & 0.868 \\
\hline N2 & $53(17.2)$ & $38(17.8)$ & $15(15.8)$ & 0.672 \\
\hline N3 & $94(30.4)$ & $52(24.3)$ & $42(44.2)$ & $<0.001$ \\
\hline \multicolumn{5}{|l|}{ pTNM stage } \\
\hline । & $102(33.0)$ & $80(37.4)$ & $22(23.2)$ & 0.014 \\
\hline$\|$ & $51(16.5)$ & $41(19.2)$ & $10(10.5)$ & 0.059 \\
\hline III & $148(47.9)$ & $88(41.1)$ & $60(63.2)$ & $<0.001$ \\
\hline IV & $8(2.6)$ & $5(2.3)$ & $3(3.2)$ & 0.975 \\
\hline
\end{tabular}


Table 2 Study population and baseline characteristics of the patients sorted by the CONUT score (Continued)

\begin{tabular}{lllll}
\hline Characteristics & All $(\boldsymbol{N}=\mathbf{3 0 9})$ & CONUT $<\mathbf{2 . 5}(\boldsymbol{N}=\mathbf{2 1 4})$ & CONUT $>\mathbf{2 . 5}(\boldsymbol{N}=\mathbf{9 5})$ & $\boldsymbol{P}$ value \\
\hline Postoperative stay, days & $13.6 \pm 0.5$ & $11.6 \pm 0.5$ & $14.1 \pm 0.7$ & 0.006 \\
Postoperative complications & $91(29.4)$ & $41(19.2)$ & $50(52.6)$ & $<0.001$ \\
\hline
\end{tabular}

Values in parentheses are percentages unless indicated otherwise; the other values are mean $\pm \mathrm{Sd}$ $B M I$ body mass index; $H b$ haemoglobin; CRP C-reactive protein; WBC white blood cells; $R B C$ red blood cells

anastomotic leakage and intra-abdominal abscess, as being related to the CONUT score. Huang et al. [39] reported that the CONUT score was a significant risk factor for total complications and 1-year survival in elderly GC patients. A meta-analysis of prognostic significance of CONUT in GC was conducted by Takagi et al., and they suggested that the preoperative CONUT score was an independent predictor of survival and postoperative complications [41]. Our results were consistent with previous studies. In our study, stratified analysis of postoperative complications was further performed to compare low and high CONUT scores. Sometimes patients suffered multiple complications. For example, after surgery, one patient suffered a sudden stomach ache and subsequent fever with abdominal tenderness and rebound tenderness as a result of duodenal

Table 3 Comparison of postoperative complications in gastric cancer undergoing laparoscopic surgery with low and high CONUT score

\begin{tabular}{|c|c|c|c|c|}
\hline Postoperative complications & All $(N=309)$ & CONUT< $2.5(N=214)$ & CONUT> $2.5(N=95)$ & $P$ value \\
\hline Overall complications & $91(29.4)$ & $41(19.2)$ & $50(52.6)$ & $<0.001$ \\
\hline Mild complications (grade I to II) & $42(13.6)$ & $17(7.9)$ & $25(26.3)$ & $<0.001$ \\
\hline Fever $>38.5^{\circ} \mathrm{C}$ after surgery & $9(2.9)$ & $4(1.9)$ & $5(5.3)$ & 0.204 \\
\hline Incision infection & $5(1.6)$ & $2(0.9)$ & $3(3.2)$ & 0.347 \\
\hline TPN $>2$ weeks & $10(3.2)$ & $4(1.9)$ & $6(6.3)$ & 0.091 \\
\hline Postoperative blood transfusion> $2 U$ & $5(1.6)$ & $2(0.9)$ & $3(3.2)$ & 0.347 \\
\hline Gastroplegia & $2(0.6)$ & $1(0.5)$ & $1(1.1)$ & 0.521 \\
\hline Early postoperative bowel obstruction & $10(3.2)$ & $4(1.9)$ & $6(6.3)$ & 0.091 \\
\hline Urinary tract infection & $1(0.3)$ & $0(0.0)$ & $1(1.1)$ & 0.307 \\
\hline Major complications (grade III to IV) & $70(22.7)$ & $40(18.7)$ & $30(31.6)$ & 0.013 \\
\hline Postoperative active haemorrhage & $16(5.2)$ & $10(4.7)$ & $6(6.3)$ & 0.548 \\
\hline Abdominal/pelvic effusion & $7(2.3)$ & $3(1.4)$ & $4(4.2)$ & 0.264 \\
\hline Intra-abdominal abscess & $17(5.5)$ & $11(5.1)$ & $6(6.3)$ & 0.676 \\
\hline Anastomotic leakage & $9(2.9)$ & $5(2.3)$ & $4(4.2)$ & 0.591 \\
\hline Anastomotic stenosis & $4(1.3)$ & $3(1.4)$ & $1(1.1)$ & 0.802 \\
\hline Duodenal stump fistula & $9(2.9)$ & $6(2.8)$ & $3(3.2)$ & 0.864 \\
\hline Septic shock & $3(1.0)$ & $0(0.0)$ & $3(3.2)$ & 0.028 \\
\hline Single organ dysfunction & $4(1.3)$ & $2(0.9)$ & $2(2.1)$ & 0.768 \\
\hline MODS & $1(0.3)$ & $0(0.0)$ & $1(1.1)$ & 0.307 \\
\hline Dead cases (grade V) & $1(0.3)$ & $0(0.0)$ & $1(1.1)$ & 0.307 \\
\hline Surgical site infection, SSI & $25(8.1)$ & $13(6.1)$ & $12(12.6)$ & 0.051 \\
\hline Surface incisional infection & $5(1.6)$ & $2(0.9)$ & $3(3.2)$ & 0.347 \\
\hline Deep space infection & $20(6.5)$ & $11(5.1)$ & $9(9.5)$ & 0.153 \\
\hline Respiratory complications & $20(6.5)$ & $8(3.7)$ & $12(12.6)$ & 0.003 \\
\hline Cardiovascular complications & $7(2.3)$ & $3(1.4)$ & $4(4.2)$ & 0.264 \\
\hline Postoperative stay, days & $13.6 \pm 0.5$ & $11.6 \pm 0.5$ & $14.1 \pm 0.7$ & 0.006 \\
\hline
\end{tabular}

Values in parentheses are percentages unless indicated otherwise; the other values are mean $\pm \mathrm{Sd}$

TPN total parenteral nutrition; ICU intensive care unit; MODS multiple organ dysfunction syndrome; SSI surgical site infection

Postoperative complications were classified from grade I to V based on the Clavien-Dindo classification system, with grade I to II defined as mild complications, grade III to IV defined as major complications 
Table 4 Univariate and multivariate analysis of risk factors associated with postoperative complications in patients with gastric cancer undergoing laparoscopic surgery

\begin{tabular}{|c|c|c|c|c|c|c|}
\hline \multirow[t]{2}{*}{ Characteristics } & \multirow{2}{*}{$\begin{array}{l}\text { Postoperative } \\
\text { complications } \\
(N=91)\end{array}$} & \multirow{2}{*}{$\begin{array}{l}\text { No postoperative } \\
\text { complications } \\
(N=218)\end{array}$} & \multirow[t]{2}{*}{$P$ value } & \multicolumn{3}{|c|}{ Multivariate } \\
\hline & & & & $\overline{\mathrm{OR}}$ & $95 \% \mathrm{Cl}$ & $P$ value \\
\hline Age, year & $68.2 \pm 1.1$ & $61.4 \pm 0.7$ & $<0.001$ & 2.237 & $1.048-4.774$ & 0.037 \\
\hline \multicolumn{7}{|l|}{ Gender } \\
\hline Male & $70(76.9)$ & $158(72.5)$ & 0.418 & & & \\
\hline Female & $21(23.1)$ & $60(27.5)$ & 0.418 & & & \\
\hline $\mathrm{BMI}, \mathrm{kg} / \mathrm{m}^{2}$ & $22.2 \pm 0.3$ & $23.0 \pm 0.2$ & 0.059 & & & \\
\hline \multicolumn{7}{|l|}{ Comorbidities } \\
\hline Diabetes mellitus & $14(15.4)$ & $24(11.0)$ & 0.286 & & & \\
\hline Hypertension & $39(42.9)$ & $78(35.8)$ & 0.242 & & & \\
\hline History of abdomen surgery & $20(22.0)$ & $50(22.9)$ & 0.855 & & & \\
\hline \multicolumn{7}{|c|}{ Preoperative laboratory measurements } \\
\hline $\mathrm{Hb}, \mathrm{g} / \mathrm{L}$ & $113.6 \pm 2.9$ & $128.2 \pm 1.4$ & $<0.001$ & 0.521 & $0.219-1.237$ & 0.139 \\
\hline $\mathrm{CRP}, \mathrm{mg} / \mathrm{L}$ & $9.0 \pm 1.8$ & $3.5 \pm 0.6$ & $<0.001$ & 1.193 & $0.500-2.849$ & 0.691 \\
\hline $\mathrm{WBC}, \times 10^{9} / \mathrm{L}$ & $5.85 \pm 0.19$ & $5.88 \pm 0.11$ & 0.881 & & & \\
\hline $\mathrm{RBC}, \times 10^{12} / \mathrm{L}$ & $3.78 \pm 0.08$ & $4.29 \pm 0.04$ & $<0.001$ & 0.356 & $0.180-0.707$ & 0.003 \\
\hline Platelets, $\times 10^{9} / \mathrm{L}$ & $216.0 \pm 7.5$ & $217.2 \pm 4.5$ & 0.887 & & & \\
\hline CONUT score & $3.7 \pm 0.3$ & $1.6 \pm 0.1$ & $<0.001$ & 2.433 & $1.218-4.862$ & 0.012 \\
\hline Albumin, g/L & $36.4 \pm 0.6$ & $40.1 \pm 0.3$ & $<0.001$ & & & \\
\hline Total lymphocytes, $\times 10^{9} / \mathrm{L}$ & $1.33 \pm 0.06$ & $1.66 \pm 0.05$ & $<0.001$ & & & \\
\hline Cholesterol, $\mathrm{mmol} / \mathrm{L}$ & $4.20 \pm 0.13$ & $4.77 \pm 0.07$ & $<0.001$ & & & \\
\hline \multicolumn{7}{|l|}{ Preoperative tumour biomarkers } \\
\hline CA125, u/ml & $15.5 \pm 1.4$ & $12.8 \pm 1.0$ & 0.129 & & & \\
\hline CA199, u/ml & $38.7 \pm 13.5$ & $23.3 \pm 3.2$ & 0.126 & & & \\
\hline CEA, ng/ml & $6.2 \pm 1.5$ & $5.6 \pm 2.2$ & 0.869 & & & \\
\hline AFP, $\mu \mathrm{g} / \mathrm{L}$ & $22.5 \pm 19.4$ & $3.3 \pm 0.3$ & 0.128 & & & \\
\hline Types of operative procedure & & & & 1.345 & $0.740-2.444$ & 0.331 \\
\hline Distal gastrectomy & $49(53.8)$ & $144(66.1)$ & 0.043 & & & \\
\hline Total gastrectomy & $42(46.2)$ & $74(33.9)$ & 0.043 & & & \\
\hline Intraoperative fluid utilisation, $\mathrm{ml}$ & $2082 \pm 66.6$ & $2195 \pm 42.1$ & 0.148 & & & \\
\hline Operative time, min & $272.7 \pm 5.3$ & $271.5 \pm 3.7$ & 0.853 & & & \\
\hline Estimated blood loss, ml & $124.2 \pm 11.8$ & $98.5 \pm 9.1$ & 0.110 & & & \\
\hline \multicolumn{7}{|l|}{ T factor } \\
\hline $\mathrm{T} 1$ & $14(15.4)$ & 77 (35.3) & $<0.001$ & 1.131 & $0.353-3.622$ & 0.836 \\
\hline $\mathrm{T} 2$ & $8(8.8)$ & $27(12.4)$ & 0.364 & & & \\
\hline T3 & $16(17.6)$ & $26(11.9)$ & 0.186 & & & \\
\hline $\mathrm{T} 4$ & $53(58.2)$ & $88(40.4)$ & 0.004 & 1.402 & $0.643-3.058$ & 0.396 \\
\hline \multicolumn{7}{|l|}{ N factor } \\
\hline NO & $25(27.5)$ & $93(42.7)$ & 0.012 & 2.596 & $0.810-8.317$ & 0.108 \\
\hline N1 & $14(15.4)$ & $30(13.8)$ & 0.710 & & & \\
\hline N2 & $10(11.0)$ & $43(19.7)$ & 0.063 & & & \\
\hline N3 & $42(46.2)$ & $52(23.9)$ & $<0.001$ & 1.903 & $0.936-3.868$ & 0.075 \\
\hline
\end{tabular}


Table 4 Univariate and multivariate analysis of risk factors associated with postoperative complications in patients with gastric cancer undergoing laparoscopic surgery (Continued)

\begin{tabular}{|c|c|c|c|c|c|c|}
\hline \multirow[t]{2}{*}{ Characteristics } & \multirow{2}{*}{$\begin{array}{l}\text { Postoperative } \\
\text { complications } \\
(N=91)\end{array}$} & \multirow{2}{*}{$\begin{array}{l}\text { No postoperative } \\
\text { complications } \\
(N=218)\end{array}$} & \multirow[t]{2}{*}{$P$ value } & \multicolumn{3}{|c|}{ Multivariate } \\
\hline & & & & $\overline{\mathrm{OR}}$ & $95 \% \mathrm{Cl}$ & $P$ value \\
\hline \multicolumn{7}{|l|}{ pTNM stage } \\
\hline । & $19(20.9)$ & $83(38.1)$ & 0.003 & 1.141 & $0.302-4.311$ & 0.846 \\
\hline$\|$ & $10(11.0)$ & $41(18.8)$ & 0.092 & & & \\
\hline III & $60(65.9)$ & $88(40.4)$ & $<0.001$ & 2.897 & $0.986-8.511$ & 0.053 \\
\hline IV & $2(2.2)$ & $6(2.8)$ & 0.780 & & & \\
\hline Postoperative stay, days & $20.7 \pm 1.4$ & $10.2 \pm 0.2$ & $<0.001$ & & & \\
\hline
\end{tabular}

Values in parentheses are percentages unless indicated otherwise; the other values are mean $\pm \mathrm{Sd}$

$B M I$ body mass index; $H b$ haemoglobin; CRP C-reactive protein; WBC white blood cells; RBC red blood cells; CONUT Controlling Nutritional Status

stump rupture, rapidly developed a serious intraabdominal abscess and had to undergo a second operation with suturing, irrigation and drainage. Our analysis indicated that a higher proportion of patients with a high CONUT score developed postoperative complications, especially mild complications. We speculated that patients with hypoalbuminemia, decreased lymphocytes and hypocholesterolaemia were more likely to experience negative conditions with slow tissue repair and delayed wound healing, increasing their susceptibility to infection, prolonging their reliance on parenteral nutrition support and increasing their probability of anastomotic complications and others. SSIs are infections of the incision, organ or nearby space that occur after surgery, which can be combined with complex comorbidities and antimicrobial-resistant pathogens, and increase the challenges and expenses of treatment [27]. There was no significant difference in SSIs located at the surface incision or deep space. The respiratory complications after surgery included pneumonia and hydrothorax, which occurred more frequently in the high CONUT score group, as reported by Song Ryo et al. [17]. We considered that long stays in bed and infrequent cough and sputum may be to blame. In summary, the CONUT score acts as an evaluation strategy for precise risk stratification of postoperative complications, which allows doctors to implement active nutritional interventions for GC patients.

Despite our findings, there were still some limitations of the present study. First, this single-centre study included a homogeneous cohort of patients with a fixed surgical team. Second, selection bias cannot be ruled out in a retrospective study. Finally, follow-up assessments of the CONUT score after surgery were not available, which resulted in a lack of dynamic observations of the nutrition status. Therefore, prospective multicentre studies are warranted to confirm the predictive significance of the CONUT score for GC patients compared with other commonly used nutritional assessments and to validate the effectiveness of preoperative nutritional interventions.

\section{Conclusion}

As a simple and feasible nutritional assessment tool, the CONUT score reliably predicts postoperative complications for patients with GC after laparoscopic gastrectomy, allowing precise risk stratification and preoperative nutritional interventions before surgery.

\section{Abbreviations}

CONUT: Controlling Nutritional Status; GC: Gastric cancer; PNI: Prognostic Nutritional Index; ALB: Albumin; PLR: Platelet-to-lymphocyte ratio;

NRS: Nutritional Risk Screening; Hb: Haemoglobin; SSI: Surgical site infection; OR: Odds ratio; TPN: Total parenteral nutrition

\section{Acknowledgements}

We thank all the authors and their family for supporting this study.

\section{Authors' contributions}

Y.Q. and H.Y.L. contributed to the manuscript writing. X.L.G., Y.Q., J.H.P., J.Q.G. and H.Y.L. contributed to date collection. W.H.Y., J.M.L. and J.F.Y. contributed to analysis of data, Y.Q. and H.Y.L. were involved in manuscript editing. X.L.G., W.Z. and X.F.W. contributed to the trial design and critical revision. The author(s) read and approved the final manuscript.

\section{Funding}

This work was supported by grants from Zhejiang Provincial Natural Science Foundation (No. 2017C33159, No. LY18H030006).

\section{Availability of data and materials}

All the data can be obtained from the author by email (gxlmed@zju.edu.cn).

\section{Ethics approval and consent to participate}

This study was approved by the ethics committee of Sir Run Run Shaw Hospital. Written informed consent was obtained from all participants.

\section{Consent for publication \\ Written informed consent was obtained from all patients enrolled in the investigation. The study protocol conformed to the ethical guidelines of the 1975 Declaration of Helsinki and the guidelines of the regional ethical committees of Sir Run Run Shaw Hospital, School of Medicine, Zhejiang University, China.}

\section{Competing interests}

No other conflict of interests.

\section{Author details}

${ }^{1}$ Department of General Surgery, Sir Run Run Shaw Hospital, School of Medicine, Zhejiang University, 3 East Qingchun Road, Hangzhou 310016, China. ${ }^{2}$ Department of Medicine, GuangXi Medical College, Nanning, China. 
Received: 25 November 2020 Accepted: 13 January 2021

Published online: 23 January 2021

\section{References}

1. Bray F, Ferlay J, Soerjomataram I, et al. Global cancer statistics 2018: GLOBOCAN estimates of incidence and mortality worldwide for 36 cancers in 185 countries. CA Cancer J Clin. 2018:68:394-424.

2. Van Cutsem E, Sagaert X, Topal B, et al. Gastric cancer. The Lancet. 2016;388: 2654-64.

3. Japanese Gastric Cancer A. Japanese gastric cancer treatment guidelines 2014 (ver. 4). Gastric Cancer. 2017;20:1-19.

4. Rinninella E, Cintoni M, Raoul P, et al. Muscle mass, assessed at diagnosis by L3-CT scan as a prognostic marker of clinical outcomes in patients with gastric cancer: a systematic review and meta-analysis. Clin Nutr. 2020;39(7):2045-54.

5. Oh SE, Choi MG, Seo JM, et al. Prognostic significance of perioperative nutritional parameters in patients with gastric cancer. Clin Nutr. 2019;38:870-6.

6. Sun X, Wang J, Liu J, et al. Albumin concentrations plus neutrophil lymphocyte ratios for predicting overall survival after curative resection for gastric cancer. Onco Targets Ther. 2016;9:4661-9.

7. Kim EY, Lee JW, Yoo HM, et al. The platelet-to-lymphocyte ratio versus neutrophil-to-lymphocyte ratio: which is better as a prognostic factor in gastric cancer? Ann Surg Oncol. 2015;22:4363-70.

8. Zheng ZF, Lu J, Xie JW, et al. Preoperative skeletal muscle index vs the controlling nutritional status score: which is a better objective predictor of long-term survival for gastric cancer patients after radical gastrectomy? Cancer Med. 2018:7:3537-47.

9. Galizia G, Lieto E, Auricchio A, et al. Naples prognostic score, based on nutritional and inflammatory status, is an independent predictor of longterm outcome in patients undergoing surgery for colorectal cancer. Dis Colon Rectum. 2017;60:1273-84.

10. Hirashima K, Watanabe $M$, Shigaki $H$, et al. Prognostic significance of the modified Glasgow prognostic score in elderly patients with gastric cancer. J Gastroenterol. 2014:49:1040-6.

11. Almasaudi AS, McSorley ST, Dolan RD, et al. The relation between Malnutrition Universal Screening Tool (MUST), computed tomographyderived body composition, systemic inflammation, and clinical outcomes in patients undergoing surgery for colorectal cancer. Am J Clin Nutr. 2019; 110(6):1327-34.

12. Li YF, Nie RC, Wu T, et al. Prognostic value of the nutritional risk screening 2002 scale in metastatic gastric cancer: a large-scale cohort study. J Cancer. 2019:10:112-9.

13. Ignacio de Ulibarri J, Gonzalez-Madrono A, de Villar NG, et al. CONUT: a tool for controlling nutritional status. First validation in a hospital population. Nutr Hosp. 2005;20:38-45.

14. Iseki Y, Shibutani M, Maeda K, et al. Impact of the preoperative controlling nutritional status (CONUT) score on the survival after curative surgery for colorectal cancer. PLoS One. 2015;10:e0132488.

15. Takagi K, Yagi T, Umeda Y, et al. Preoperative controlling nutritional status (CONUT) score for assessment of prognosis following hepatectomy for hepatocellular carcinoma. World J Surg. 2017:41:2353-60.

16. Toyokawa T, Kubo N, Tamura T, et al. The pretreatment controlling nutritional status (CONUT) score is an independent prognostic factor in patients with resectable thoracic esophageal squamous cell carcinoma: results from a retrospective study. BMC Cancer. 2016:16:722

17. Ryo S, Kanda M, Ito S, et al. The controlling nutritional status score serves as a predictor of short- and long-term outcomes for patients with stage 2 or 3 gastric cancer: analysis of a multi-institutional data set. Ann Surg Oncol. 2019;26:456-64

18. Lin JX, Lin LZ, Tang YH, et al. Which nutritional scoring system is more suitable for evaluating the short- or long-term prognosis of patients with gastric cancer who underwent radical gastrectomy? J Gastrointest Surg. 2020;24(9):1969-77.

19. Hirahara N, Tajima Y, Fujii Y, et al. Controlling Nutritional Status (CONUT) as a prognostic immunonutritional biomarker for gastric cancer after curative gastrectomy: a propensity score-matched analysis. Surg Endosc. 2019;33(12): 4143-52.

20. Liu X, Zhang D, Lin E, et al. Preoperative controlling nutritional status (CONUT) score as a predictor of long-term outcome after curative resection followed by adjuvant chemotherapy in stage II-III gastric Cancer. BMC Cancer. 2018;18:699.
21. Kuroda D, Sawayama H, Kurashige J, et al. Controlling Nutritional Status (CONUT) score is a prognostic marker for gastric cancer patients after curative resection. Gastric Cancer. 2018;21:204-12.

22. Abdikarim I, Cao XY, Li SZ, et al. Enhanced recovery after surgery with laparoscopic radical gastrectomy for stomach carcinomas. World Gastroenterol. 2015;21:13339-44.

23. Kang SH, Lee $\mathrm{Y}, \mathrm{Min} \mathrm{SH}$, et al. Multimodal enhanced recovery after surgery (ERAS) program is the optimal perioperative care in patients undergoing totally laparoscopic distal gastrectomy for gastric cancer: a prospective, randomized, clinical trial. Ann Surg Oncol. 2018;25:3231-8.

24. Tweed T, van Eijden Y, Tegels J, et al. Safety and efficacy of early oral feeding for enhanced recovery following gastrectomy for gastric cancer: a systematic review. Surg Oncol. 2019;28:88-95.

25. Dindo D, Demartines N, Clavien PA. Classification of surgical complications: a new proposal with evaluation in a cohort of 6336 patients and results of a survey. Ann Surg. 2004;240:205-13

26. Ge X, Liu H, Tang S, et al. Preoperative hypoalbuminemia is an independent risk factor for postoperative complications in Crohn's disease patients with normal BMl: a cohort study. Int J Surg. 2020;79:294-9.

27. Berrios-Torres SI, Umscheid CA, Bratzler DW, et al. Centres for disease control and prevention guideline for the prevention of surgical site infection, 2017. JAMA Surg. 2017:152:784-91.

28. Gioulbasanis I, Martin L, Baracos VE, et al. Nutritional assessment in overweight and obese patients with metastatic cancer: does it make sense? Ann Oncol. 2015;26:217-21.

29. Arends J, Bachmann P, Baracos $V$, et al. ESPEN guidelines on nutrition in cancer patients. Clin Nutr. 2017;36:11-48.

30. Hargadon KM. Tumour microenvironmental influences on dendritic cell and T cell function: a focus on clinically relevant immunologic and metabolic checkpoints. Clin Transl Med. 2020;10:374-411.

31. Saka D, Gokalp M, Piyade B, et al. Mechanisms of T-cell exhaustion in pancreatic cancer. Cancers (Basel). 2020;12(8):2274.

32. Yang Z, Qin W, Chen Y, et al. Cholesterol inhibits hepatocellular carcinoma invasion and metastasis by promoting CD44 localization in lipid rafts. Cancer Lett. 2018:429:66-77.

33. Park SH, Lee $\mathrm{S}$, Song $\mathrm{JH}$, et al. Prognostic significance of body mass index and prognostic nutritional index in stage II/II gastric cancer. Eur J Surg Oncol. 2020;46:620-5.

34. Jiang N, Deng JY, Ding XW, et al. Prognostic nutritional index predicts postoperative complications and long-term outcomes of gastric cancer World J Gastroenterol. 2014;20:10537-44.

35. Hirahara N, Tajima Y, Fujii Y, et al. Prognostic nutritional index as a predictor of survival in resectable gastric cancer patients with normal preoperative serum carcinoembryonic antigen levels: a propensity score matching analysis. BMC Cancer. 2018;18:285.

36. Sasahara M, Kanda M, Ito S, et al. The preoperative prognostic nutritional index predicts short-term and long-term outcomes of patients with stage II/III gastric cancer: analysis of a multi-institution dataset. Dig Surg. 2020;37(2):135-44.

37. Sun X, Liu X, Liu J, et al. Preoperative neutrophil-to-lymphocyte ratio plus platelet-to-lymphocyte ratio in predicting survival for patients with stage $1-||$ gastric cancer. Chin J Cancer. 2016;35(1):57.

38. Inaoka K, Kanda M, Uda H, et al. Clinical utility of the platelet-lymphocyte ratio as a predictor of postoperative complications after radical gastrectomy for clinical T2-4 gastric cancer. World J Gastroenterol. 2017;23:2519-26.

39. Huang Y, Huang Y, Lu M, et al. Controlling Nutritional Status (CONUT) score is a predictor of post-operative outcomes in elderly gastric cancer patients undergoing curative gastrectomy: a prospective study. Cancer Manag Res. 2019;11:9793-800.

40. Li Y, Tan B, Fan L, et al. Clinicopathologic characteristics of elderly with gastric cancer, and the risk factors of postoperative complications. J Invest Surg. 2017:30:394-400

41. Takagi K, Domagala P, Polak WG, et al. Prognostic significance of the controlling nutritional status (CONUT) score in patients undergoing gastrectomy for gastric cancer: a systematic review and meta-analysis. BMC Surg. 2019;19:129.

\section{Publisher's Note}

Springer Nature remains neutral with regard to jurisdictional claims in published maps and institutional affiliations. 